\title{
Tradução e Validação do Perceived Autonomy Support: Exercise Climate Questionnaire numa Amostra de Praticantes de Exercício Portugueses
}

\section{Translation and Validation of the Perceived Autonomy Support: Exercise Climate Questionnaire in a Sample of Portuguese Exercisers}

\author{
João Miguel Raimundo Peres Moutão*, ${ }^{*}$, Luís Cid $^{a}$, José Carlos Leitão ${ }^{b} \&$ José Alves $^{c}$ \\ ${ }^{a}$ Escola Superior de Desporto de Rio Maior - Instituto Politécnico de Santarém, Santarém, Portugal, \\ ${ }^{b}$ Universidade de Trás-os-Montes e Alto Douro, Vila Real, Portugal \\ $\&{ }^{c}$ Cooperativa de Ensino Superior Politécnico e Universitário Norte, Gandra, Portugal
}

\begin{abstract}
Resumo
O objetivo do presente estudo foi o de traduzir e avaliar as propriedades psicométricas do Perceived Autonomy Support Exercise Climate Questionnaire, para avaliar a perceção do suporte de autonomia dado pelo professor de fitness, em linha com a teoria da autodeterminação. Para este propósito, recorreu-se a uma amostra de 935 praticantes de exercício, masculinos $(N=344)$ e femininos $(N=591)$, pertencentes a 16 academias da zona de Lisboa e litoral oeste de Portugal. As análises efetuadas revelaram que a versão traduzida possuía uma adequada validade interna, validade temporal, validade de construto e validade preditiva, dando sustentação à sua utilização em praticantes de exercício Portugueses.

Palavras-chave: Suporte da autonomia, teoria da autodeterminação, psicometria.
\end{abstract}

\begin{abstract}
The aim of the present study was to translate and evaluate the psychometric properties of the Perceived Autonomy Support: Exercise Climate Questionnaire that measures the autonomy support given by fitness instructors in accordance with the self-determination theory. In order to fulfill such purpose, it was collected a sample of 935 fitness exercisers, male $(N=344)$ and female $(N=591)$ in 16 different Health Centers in the area of Lisbon and west coast of Portugal. The analysis performed revealed that the translated version had an adequate internal, temporal, construct and predictive validity, supporting its use with Portuguese exercisers.

Keywords: Autonomy support, self-determination theory, psychometrics.
\end{abstract}

A teoria da autodeterminação TAD (Deci \& Ryan, 1985, 2002) é uma macro teoria motivacional onde o contexto social assume um papel central. Esta importância baseiase no pressuposto de que, como clarificam Deci e Ryan (1991), toda a atividade humana ocorre integrada num contexto social, seja ele real, por estarem fisicamente presentes outras pessoas, ou imaginado, por inconscientemente agirmos de forma como achamos que outras pessoas gostariam que nos comportássemos.

De acordo com a TAD, um ambiente social que suporte a autonomia através da interação com as "pessoas chave" (e.g. professor, amigos e familiares), irá promover a satisfação das necessidades psicológicas básicas (NPB) de autonomia, competência e relação (Ryan \& Deci, 2006).

\footnotetext{
*Endereço para Correspondência: Instituto Politécnico de Santarém, Escola Superior de Desporto de Rio Maior, Av. Dr. Mário Soares, Pavilhão Multiusos, Rio Maior, Portugal 2040-413. E-mail: jmoutao@esdrm.ipsantarem.pt
}

O suporte de autonomia relaciona-se assim com a provisão de opções de escolha e de um racional teórico, à consideração da perspetiva dos outros e à minimização da pressão, por parte de quem está numa posição de liderança (Deci, Eghrari, Patrick, \& Leone, 1994). É exemplo disso um instrutor de fitness que tem em consideração a perspetiva dos praticantes e incentiva-os a tomarem as suas próprias decisões, ao invés de impor o seu ponto de vista fazendo com que sejam os praticantes de exercício a terem de se adaptar às suas ideias (Edmunds, Duda, \& Ntoumanis, 2008).

A importância do suporte da autonomia para a satisfação das necessidades psicológicas básicas, e consequentes benefícios comportamentais, cognitivos e afetivos, tem sido evidenciada em contextos tão distintos como o do ensino (Reeve, 2002; Reeve, Jang, Carrell, Jeon, \& Barch, 2004; Vansteenkiste, Simons, Lens, Sheldon, \& Deci, 2004) e o familiar (Mireille, Renée, \& Richard, 2008), bem como em variados comportamentos de saúde (Ryan, Patrick, Deci, \& G. C. Williams, 2008), de que são exem- 
plo a tomada de medicação contra o HIV (Kennedy, Goggin, \& Nollen, 2004), a cessação tabágica (G. C. Williams et al., 2006) e o controlo da diabetes (G. C. Williams et al., 2009) e da glicémia (G. C. Williams, McGregor, King, Nelson, \& Glasgow, 2005).

Também no contexto desportivo, e em linha com os pressupostos teóricos da TAD, um substancial volume de investigação tem demonstrado que quando o Treinador promove a autonomia dos atletas, é promovida a satisfação das NPB, a regulação autónoma, a adesão e vitalidade (Adie, Duda, \& Ntoumanis, 2008; Álvarez, Balaguer, Castillo, \& Duda, 2009; Amorose \& Anderson-Butcher, 2007; Balaguer, Castillo, \& Duda, 2008b; Gagne, Ryan, \& Bargmann, 2003; Reinbotha \& Duda, 2006). No que ao contexto do exercício diz respeito, o recurso habitual às avaliações e classificações, bem como a utilização de quadros de honra e os elogios públicos, poderão acrescentar alguma pressão social aos praticantes, com claros prejuízos ao nível da sua motivação intrínseca e estado emocional. Não é assim de admirar que o suporte de autonomia dado pelos professores de fitness tenha sido relacionado com mais motivação intrínseca, adesão e saúde emocional (Edmunds, Ntoumanis, \& Duda, 2006, 2007b; Wilson \& Rodgers, 2004), bem como uma melhoria dos estados emocionais pós-exercício de vitalidade e tranquilidade (Vlachopoulos \& Triantafyllidou, 2008).

A importância do suporte de autonomia em praticantes de exercício foi evidenciada num estudo experimental e longitudinal (10 semanas) realizado por Edmunds et al. (2008). Nesta investigação duas classes de exercício foram alvo de diferentes estilos de ensino por parte do mesmo professor (i.e. estilo de ensino de "suporte da autonomia" e estilo de ensino "tradicional"). O suporte de autonomia foi avaliado simultaneamente através do questionário de auto-perceção Perceived Environmental Supportiveness Scale (Tobin, 2003) e de uma escala de observação adaptada de Reeve et al. (2004) preenchida por observadores independentes. Os resultados demonstraram que na classe em que o professor utilizou um estilo de ensino de "suporte da autonomia", aumentaram linearmente, ao longo das 10 semanas, a perceção de suporte de autonomia, a satisfação das necessidades psicológicas básicas, a autodeterminação, a adesão e a melhoria do estado de humor. Os benefícios do suporte da autonomia têm também sido evidenciados em programas de exercício destinados a populações especiais, como praticantes obesos (Edmunds, Ntoumanis, \& Duda, 2007a; Silva et al., 2011; Silva et al., 2010) e doentes coronários (Kubitz, 2010).

Estes estudos permitem assumir com alguma segurança o papel promissor que a TAD pode assumir na explicitação do processo pelo qual o ambiente social influência a prática de exercício físico, pondo a descoberto o papel decisivo dos professores de fitness, na medida em que são estes que orientam as sessões de exercício e dão suporte à autonomia do praticante, demonstrando interesse, dedicando recursos, dando apoio e feedback constante, com base numa relação de confiança e afetividade. Ainda que, como analisaram Murcia, Roman, Galindo, Alonso e Gonzalez (2008), o clima motivacional gerado pelos "outros praticantes", através da ênfase na cooperação e na melhoria pessoal, possa igualmente contribuir para a satisfação das necessidades psicológicas básicas, aumentando assim a autodeterminação para a prática de exercício.

A possibilidade de modificação do contexto social assume uma maior relevância porquanto, como referem Edmunds, Ntoumanis e Duda (2007b), este se constitui como o fator que mais facilmente poderá ser alvo de modificação por parte dos professores. Dessa forma a existência de um instrumento para a avaliação deste construto em praticantes de exercício portugueses, reveste-se de um maior interesse na medida em que ambientes sociais percecionados como "controladores" relacionam-se com sentimentos de aborrecimento, desinteresse e infelicidade, podendo levar ao abandono e à falta de persistência na prática de exercício (Kowal \& Fortier, 2000; Pelletier, Fortier, Vallerand, \& Brière, 2001), sendo esta problemática importante no contexto do exercício físico em academias. Assim sendo, o objetivo principal deste estudo é o da tradução, adaptação e validação da versão original do questionário PASECQ.

\section{Metodologia}

\section{Amostra}

A amostra foi constituída por 935 praticantes de fitness, do sexo feminino $(N=591 ; 63,2 \%)$ e masculino $(N=$ $344 ; 36,8 \%$ ), com idades compreendidas entre os 16 e os 70 anos $(M=32,70 ; D P=12,29)$, envolvidos nas modalidades de cardiofitness $(N=233 ; 24,9 \%)$, musculação $(N=266 ; 28,4 \%)$ e aulas de grupo $(N=436 ; 46,6 \%)$. A frequência semanal de prática de exercício variou entre 1 a 6 vezes por semana $(M=3,1, D P=1,1)$ a que correspondeu uma duração de prática semanal entre $1 \mathrm{a}$ 15 horas $(M=4,2, D P=2,4)$. No que diz respeito ao tempo de prática, 337 praticantes (36\%) frequentavam a academia há menos de 6 meses, 227 praticantes $(24,3 \%)$ entre 6 e 18 meses sendo que os restantes 371 praticantes $(39,7 \%)$ praticavam exercício continuamente há mais de 18 meses.

\section{Instrumentos de Medida}

O Perceived Autonomy Support: Exercise Climate Questionnaire (PASECQ) consiste num instrumento de autorrelato adaptado ao contexto do exercício físico por Edmunds et al. (2006) a partir da versão original do Perceived Autonomy Support: Health Care Climate Questionnaire (PASHCCQ; G. C. Williams, Grow, Freedman, Ryan, \& Deci, 1996). Este questionário é constituído por 6 itens, que concorrem para um único fator que avalia a perceção do suporte de autonomia dado pelo professor de fitness. A resposta é dada numa escala tipo 
likert de 1-7, correspondendo a opção "Discordo Totalmente" ao valor 1 e o "Concordo Totalmente" ao valor 7 .

Para o estudo de validade preditiva recorreu-se à versão portuguesa do questionário Basic Psychological Needs in Exercise Scale (BPNESp) para avaliar a satisfação das necessidades psicológicas básicas. O BPNES é um instrumento de autorrelato constituído por 12 itens, distribuídos pelos fatores de autonomia, competência e relação, desenvolvido especificamente para o contexto de exercício físico por Vlachopoulos e Michailidou (2006). As respostas são dadas numa escala tipo likert de $1-5$, correspondendo a opção "Discordo Totalmente" ao valor " 1 " e o extremo oposto "Concordo Totalmente" ao valor "5". Vários estudos têm suportado a fiabilidade e validade do BPNES em praticantes de exercício na Grécia (Vlachopoulos, 2007, 2008; Vlachopoulos \& Michailidou, 2006; Vlachopoulos \& Neikou, 2007), em Inglaterra (Vlachopoulos, Ntoumanis, \& Smith, 2010) e Espanha (Sánchez \& Núñez, 2007). Para a versão portuguesa aqui aplicada foram encontrados índices de ajustamento adequados $\left(\mathrm{S}-\mathrm{B} \chi^{2}=144,14 ; p=0,001 ; \chi^{2} / g l=2,82 ; \mathrm{NNFI}=\right.$ 0,938 ; CFI $=0,952$; RMSEA $=0,059 ; 90 \% \mathrm{CI}=0,048$ 0,071 ) e de consistência interna superiores a 0,07 para todas as subescalas (Moutão, Cid, Leitão, Alves, \& Vlachopoulos, 2012).

\section{Procedimentos de Recolha dos Dados}

Para a recolha dos dados foram contactados os responsáveis de 16 academias da zona centro e da área metropolitana de Lisboa, de forma a alargar o espectro mostral a academias de diferentes zonas geográficas (i.e. urbana/ rural) e dimensão (i.e. grande/familiar). Após a obtenção de autorização prévia para a realização deste estudo, por parte dos responsáveis das academias, foram feitas diversas deslocações às instalações, em diferentes horas do dia (manhã, tarde e noite) e dias da semana. Os sujeitos foram abordados de forma informal, antes de iniciarem a sua sessão de exercício. A entrega dos questionários foi sempre realizada sob a supervisão de assistentes devidamente treinados para o efeito, com base num protocolo previamente definido e após o consentimento informado dos sujeitos. Os questionários foram preenchidos individualmente, despendendo-se entre 8 a 12 minutos no seu preenchimento.

\section{Procedimentos de Tradução}

No sentido de garantir a qualidade da tradução e adaptação do questionário PASECQp, foram adotados os procedimentos metodológicos de tradução-retroversão, propostos por Banville, Desrosiers e Genet-Volet (2000) e Vallerand (1989). Dessa forma, o PASECQp foi inicialmente traduzido, de forma independente, por 4 tradutores bilingues (fase1). Após esta tradução foi constituído um painel de quatro avaliadores bilingues, com experiência académica de intervenção no contexto do exercício físico, que compararam o conteúdo da versão traduzida com a original, sugerindo ajustamentos (fase 2). Segui- damente foi constituído um novo painel de 4 avaliadores, mestres e doutorados, com experiência de intervenção na área do desporto e de investigação na área da psicologia do desporto que, após consenso, confirmaram a equivalência entre as versões original e traduzida, produzindo assim a versão inicial do PASECQp (fase 3). Esta versão foi aplicada a uma amostra de 50 alunos do ensino superior, praticantes de exercício, que revelaram uma correta compreensão do modo de preenchimento dos questionários e do significado dos itens (fase 4). Por último, dois professores de língua portuguesa fizeram uma revisão final onde garantiram a adequada aplicação da língua Portuguesa (fase 5).

\section{Procedimentos para a Análise Fatorial Confirmatória (AFC)}

Para a avaliação do ajustamento global do modelo original do PASECQ foi realizada uma análise fatorial confirmatória (AFC) através do método de estimação da máxima verosimilhança (ML) e o teste estatístico de $\chi^{2}$. No entanto, a sensibilidade o valor de $\chi^{2}$ à dimensão da amostra e distribuição das variáveis faz com que este critério seja demasiado exigente para a contrastação de modelos, especialmente em estudos nas ciências sociais onde há muitas fontes de variabilidade (Byrne, 2006). Por essa razão, serão igualmente analisados os valores obtidos nos índices alternativos de bondade de ajustamento (Hair, Black, Babin, \& Anderson, 2009; Kahn, 2006; Worthington \& Whittaker, 2006), designadamente: Comparative Fit Index (CFI; Bentler, 1990), Non-normed Fit Index (NNFI), Standardized Root Mean Residual (SRMS) e o Root Mean Square Error of Approximation (RMSEA; Steiger \& Lind, 1980) e respetivo intervalo de confiança (IC) a $90 \%$. Os valores de corte assumidos para cada um destes indicadores foram os propostos por $\mathrm{Hu}$ e Bentler (1999): $<0,08$ para o SRMR, $>0,95$ para CFI e NNFI e $<0,06$ para o RMSEA.

Considerando que a teoria subjacente ao método de estimação ML assume a multinormalidade dos itens (Kahn, 2006; Kline, 2005) será necessário recorrer ao teste de Mardia (1970) para se avaliar este pressuposto. Caso o valor estandardizado de Mardia seja superior a 5 (Byrne, 2006), será utilizado o método robusto, proposto por Satorra e Bentler (2001) que corrige os valores do $\chi^{2} \mathrm{e}$ dos índices de ajustamento para a não normalidade, apresentando-se assim o valor de Satorra-Bentler $\chi^{2}\left(\mathrm{~S}-\mathrm{B} \chi^{2}\right)$, as estimativas robustas de NNFI, CFI, RMSEA e respetivo intervalo de confiança (IC) a 90\%. Para a realização das análises fatoriais confirmatórias foi utilizado o programa estatístico EQSWIN (versão 6.1). Para identificar a qualidade do ajustamento local do modelo, foram ainda analisadas eventuais inconsistências nas estimativas dos diferentes parâmetros através da estatística descritiva das variáveis observáveis incluídas nos modelos de estrutura de covariância (i.e. média, desvio-padrão, assimetria, achatamento), do valor de fiabilidade alfa de Cronbach, bem como dos pesos fatoriais ou valores estimados 
estandardizados de cada item no seu fator e respetivas correlações bilaterais produto momento de Pearson.

A presença de outliers multivariados foi identificada pela distância quadrada de Mahalanobis, que representa "a distância de uma observação à média de todas as observações de todas as variáveis, designada por centróide" (Maroco, 2010, p. 65). Conforme sugerido por Maroco (2010), foram eliminados todos os sujeitos com $p_{1}$ e $p_{2}$ inferiores a 0,05 .

\section{Resultados}

\section{Estatística Descritiva do PASECQP}

Da leitura da Tabela 1, constata-se que os valores obtidos evidenciam médias na pontuação dos itens superiores ao valor intermédio da respetiva distribuição $(3,5$ pontos na escala de 1 a 7), podendo significar que os sujeitos da amostra percecionam o contexto social como sendo promotor, em certa medida, da sua autonomia.

Tabela 1

Estatística Descritiva Para o Modelo PASECQP

\begin{tabular}{lccccc}
\hline Escala $/$ item & Mín-Máx & Média & $D P$ & Assimetria & Curtose \\
\hline Suporte da autonomia & $2-7$ & 5,69 & 0,85 & $-0,13$ & $-0,30$ \\
Item 1 & $1-7$ & 5,48 & 1,15 & $-0,83$ & 1,85 \\
Item 2 & $2-7$ & 5,72 & 0,97 & $-0,15$ & $-0,71$ \\
Item 3 & $1-7$ & 5,56 & 1,04 & $-0,24$ & $-0,33$ \\
Item 4 & $3-7$ & 6,19 & 0,95 & $-0,83$ & $-0,26$ \\
Item 5 & $1-7$ & 5,66 & 1,11 & $-0,57$ & 0,31 \\
Item 6 & $1-7$ & 5,53 & 1,16 & $-0,42$ & $-0,19$ \\
\hline
\end{tabular}

Nota. Mín-Máx (valor mínimo e máximo); DP (desvio-padrão).

Devido a esta tendência os dados apresentam uma distribuição univariada não normal, conforme se pode verificar pelo valor das medidas de assimetria (skewness) e achatamento (kurtosis), as quais se situam fora do intervalo $<0,5 \mathrm{e}>-0,5$ para a maioria dos itens (Bulmer, 1965).

\section{Validade de Construto do Ponto de Vista Confirmatório}

Para avaliar a validade de construto do PASECQp recorreu-se a uma AFC do modelo original que assenta nos seguintes pressupostos: (a) as respostas dos sujeitos são explicadas por um único fator (suporte da autonomia), (b) a carga fatorial exibida por cada um dos itens está associada ao fator que é suposto medir (denominada carga fatorial alvo) e (c) não há correlação entre os erros de estimativa associados a cada um dos itens. Os índices de bondade de ajustamento global obtidos para este modelo indicam um adequado ajustamento global à matriz de covariância dos dados dos sujeitos da nossa amostra $\left(\mathrm{S}-\mathrm{B} \chi^{2}=20,46 ; p=0,015 ; \chi^{2} / g l=2,28 ; \mathrm{NNFI}=0,986\right.$; $\mathrm{CFI}=0,992$; RMSEA $=0,049$ com $90 \% \mathrm{CI}=0,021-$ $0,078)$. De igual forma é possível verificar, através da Tabela 2, que não existem parâmetros que exibam estimativas inadequadas como variâncias negativas ou erros de estimativa maiores que " 1 ".

Tabela 2

Parâmetros Estimados, Consistência Interna e Correlação Item-Fator para o Modelo PASECQp

\begin{tabular}{lccccc}
\hline Itens & Peso fatorial & $\begin{array}{c}\text { Erro de } \\
\text { medida }\end{array}$ & $\begin{array}{c}\text { Variância explicada } \\
\text { pelo fator }\end{array}$ & $\begin{array}{c}\alpha \\
\text { s/item }\end{array}$ & $\begin{array}{c}\text { Correlação } \\
\text { item/fator }\end{array}$ \\
\hline Item 1 & 0,72 & 0,69 & 0,49 & 0,88 & 0,77 \\
Item 2 & 0,82 & 0,58 & 0,68 & 0,86 & 0,84 \\
Item 3 & 0,80 & 0,60 & 0,63 & 0,87 & 0,82 \\
Item 4 & 0,62 & 0,78 & 0,47 & 0,88 & 0,73 \\
Item 5 & 0,75 & 0,66 & 0,61 & 0,86 & 0,83 \\
Item 6 & 0,79 & 0,62 & 0,59 & 0,87 & 0,82 \\
\hline
\end{tabular}

Nota. Todas as cargas fatoriais dos itens e as correlações item/fator são estatisticamente significativas $(p<0,05)$.

No que diz respeito às cargas fatoriais estandardizadas, verifica-se que todas são significativas $(p<0,01)$ e superiores a 0,5 , dando suporte à assunção da validade convergente dos itens nas respetivas escalas. Verifica-se igualmente que todos os itens possuem uma relação significa- tiva e forte com o fator a que pertencem. As correlações múltiplas quadradas, representando a proporção de variância explicada pelas variáveis preditoras (Byrne, 2006, 2009), exibem uma variância explicada que oscila entre $R^{2}=0,68$ e $R^{2}=0,83$. Na Figura 1 é possível a observação 
do modelo testado (PASECQp) com as respetivas cargas fatoriais estandardizadas e valores de variância explicada.

\section{Validade Interna}

No que diz respeito à consistência interna, verificou-se que versão traduzida do PASECQ apresenta um valor de alfa de Cronbach de 0,89 , claramente superior ao valor de 0,70 proposto por Nunnally (1978) como sendo aceitável. Constata-se também que este valor de alfa não poderia ser elevado a partir da eliminação de itens ( $\alpha$ escala s/item).

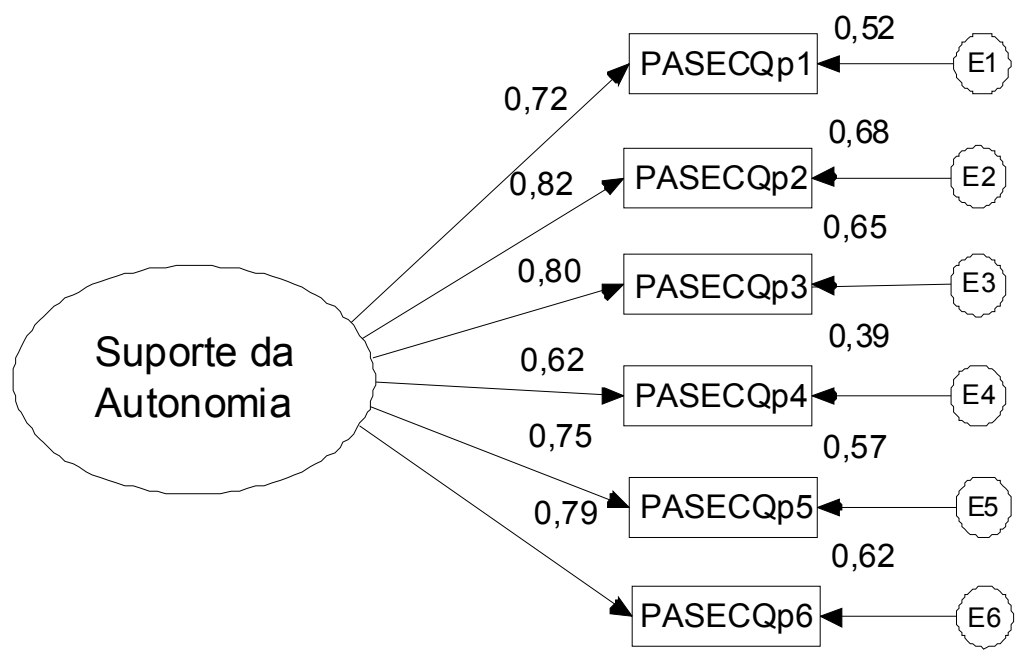

Figura 1. Solução estandardizada do modelo testado PASECQp.

Nota: PASECQp 1-6 = Número dos itens; E = erro de medida para cada item. Os valores no canto superior dos retângulos representam a proporção da variância do item que é explicada pelo respetivo fator (i.e. Squared)Multiple Correlation); os valores nas setas de ligação representam as cargas fatoriais estandardizadas dos itens.

\section{Validade Temporal}

Para avaliar a validade temporal do PASECQp, foi solicitado a 60 praticantes de exercício que preenchessem o questionário em dois momentos distintos, separados no tempo por 2 semanas. A estabilidade temporal foi calculada através do coeficiente de correlação intraclasse (CCI; Vincent, 1995), sendo que CCI superiores a 0,90 são reveladores de uma elevada estabilidade temporal. Os resultados evidenciaram que a média da perceção de autonomia mudou de 5,25 $(D P=0,83)$ para 5,72 $(D P=0,91)$ com um CCI de 0,93, encontrando-se claramente acima do valor de 0,90 proposto por Vincent (1995).

\section{Validade Preditiva}

Para analisar a validade preditiva do PASECQp foi realizada uma análise de equações estruturais que analisa o ajustamento de um modelo no qual o suporte de autonomia tem um efeito direto sobre a satisfação de cada uma das NPB de autonomia, competência e relação. Considerando que o coeficiente normalizado de Mardia foi de 53,65 , os indicadores obtidos através do método robusto, proposto por Satorra e Blenter (2001), revelaram um ajustamento adequado para este modelo ( $\mathrm{S}-\mathrm{B} \chi^{2}=385,81 ; p$ $=0,000 ; \chi^{2} / g l=2,92 ; \mathrm{NNFI}=0,920 ; \mathrm{CFI}=0,931$; RMSEA $=0,061(90 \% \mathrm{CI}=0,054-0,068)$, sendo que todos os parâmetros estimados foram significativos $(p<$ $0,01)$. Conforme se pode verificar na Figura 2, o efeito do suporte de autonomia sobre as NPB foi de 0,72 para a autonomia (Distúrbio $=0,691 ; 52 \%$ da variância explicada pelo suporte de autonomia), 0,64 para competência (Distúrbio $=0,769 ; 41 \%$ da variância explicada pelo suporte de autonomia) e 0,60 para relação (Distúrbio $=0,803$; $36 \%$ da variância explicada suporte de autonomia).

\section{Discussão}

$\mathrm{O}$ estudo realizado possibilitou o aprofundamento do conhecimento sobre a validade de construto do PASECQp, ao mesmo tempo que permitiu uma análise mais pormenorizada dos seus itens e do seu contributo individual para a avaliação deste construto. Globalmente os resultados demonstraram que o PASECQp apresenta valores adequados de fiabilidade e validade. No que diz respeito à validade de construto, os resultados da $\mathrm{AFC}$ revelaram um adequado ajustamento da estrutura unidimensional, com índices de ajustamento similares aos obtidos em validações anteriores (Álvarez et al., 2009; Balaguer, Castillo, \& Duda, 2008a; Reinboth \& Duda, 2006). No que se refere à validade preditiva do PASECQp os resultados demonstraram que o suporte de autonomia influencia positivamente a satisfação das NPB, conforme postulado pela TAD (Deci \& Ryan, 1985, 2002). De igual forma foram encontrados valores adequados de estabilidade temporal e fiabilidade interna. Os valores de alfa encontrados não poderiam ser elevados a partir da eliminação de itens e são semelhantes aos obtidos noutros estudos realizados com o PASECQ, tanto na versão longa (Balaguer et al., 2008b; G. C. Williams et al., 1996), 


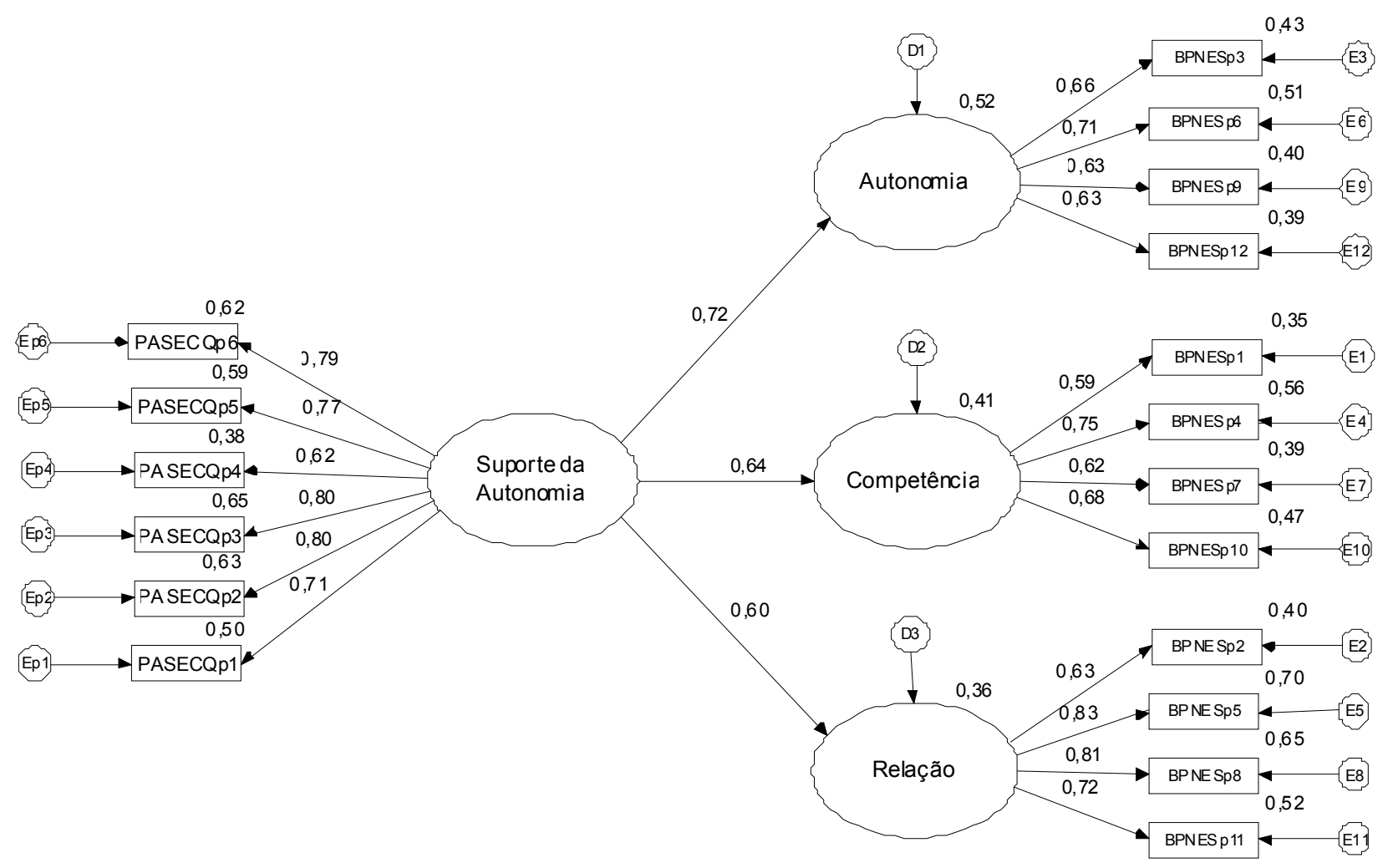

Figura 2. Modelo de equações estruturais em que se analisa o efeito do suporte de autonomia sobre a satisfação das necessidades psicológicas básicas.

Nota: PASECQp 1-6 = Número dos itens do PASECQp; BPNESp 1-12= Número dos itens do BPNESp; E = erro de medida para cada item; D 1-3 = Distúrbio ou erro de medida para cada fator. Os valores no canto superior dos retângulos representam a proporção da variância do item que é explicada pelo respetivo fator (i.e. Squared Multiple Correlation); os valores nas setas de ligação representam as cargas fatoriais estandardizadas dos itens.

como reduzida (Edmunds et al., 2007a; Vlachopoulos \& Triantafyllidou, 2008).

Por fim, no que diz respeito à validade preditiva, verificou-se que o modelo de equações estruturais, que estabelecia relações diretas entre o suporte de autonomia e a satisfação das NPB, demonstrou-se ajustado, sendo todas as relações positivas e significativas $(p<0,01)$. Estes resultados estão de acordo com os pressupostos da TAD, a qual sugere que o suporte de autonomia dado pelo contexto social influência direta e positivamente a satisfação das NPB.

Em suma, a análise às características psicométricas do questionário PASECQp demonstrou resultados adequados de validade interna, validade temporal, validade de construto e validade preditiva, dando suporte à utilização do PASECQp para a avaliação da perceção de suporte de autonomia em praticantes de exercício Portugueses. Ainda assim, entendendo-se que a validação de um instrumento é um processo dinâmico e contínuo (Messick, 1995), considera-se como preliminar este estudo de tradução, adaptação e validação. Dessa forma, será pertinente prosseguir com investigação futura sobre as qualidades psicométricas do PASECQp com vista ao seu refinamento, replicando as análises aqui realizadas com outras amostras. Também será importante realizar estudos de invariância métrica - importantes no desenvolvimento de novos instrumentos de avaliação, de forma a testar a estabilidade da estrutura do PASECQp entre grupos de sujeitos de ambos os sexos, de diferentes níveis etários e de diferentes contextos de prática.

\section{Referências}

Adie, J. W., Duda, J. L., \& Ntoumanis, N. (2008). Autonomy support, basic need satisfaction and the optimal functioning of adult male and female sport participants: A test of basic needs theory. Motivation and Emotion, 32(3), 189-199.

Álvarez, M. S., Balaguer, I., Castillo, I., \& Duda, J. L. (2009). Coach autonomy support and quality of sport engagement in young soccer players. The Spanish Journal of Psychology, 12(1), 138-148.

Amorose, A. J., \& Anderson-Butcher, D. (2007). Autonomysupportive coaching and selfdetermined motivation in high school and college athletes: A test of selfdetermination theory. Psychology of Sport and Exercise, 8(5), 654-670.

Balaguer, I., Castillo, I., \& Duda, J. L. (2008a). Apoyo a la autonomía, satisfacción de las necesidades, motivación y bienestar en deportistas de competición: un análisis de la teoría de la autodeterminación. Revista de Psicología del Deporte, 17, 123-139. 
Balaguer, I., Castillo, I., \& Duda, J. L. (2008b). Autonomy support, needs satisfaction, motivation and well-being in competitive athletes: A test of the Self-determination theory. Revista de Psicologia del Deporte, 17, 1-4.

Banville, D., Desrosiers, P., \& Genet-Volet, Y. (2000). Translating questionnaires and inventories using a cross-cultural translation technique. Journal of Teaching in Physical Education, 19, 374-387.

Bentler, P. M. (1990). Comparative fit indices in structural models. Psychological Bulletin, 107, 238-246.

Bulmer, M. G. (1965). Principles of Statistics. Cambridge, MA: Massachusetts Institute of Technology Press.

Byrne, B. (2006). Structural equation modeling with EQS: Basic concepts, applications, and programming (2 $2^{\text {nd }} \mathrm{ed}$.). Mahwah, NJ: Lawrence Erlbaum.

Byrne, B. (2009). Structural equation modeling with AMOS: Basic concepts, applications, and programming ( $2^{\text {nd }}$ ed.). New York: Routledge Academic.

Deci, E. L., Eghrari, H., Patrick, B. C., \& Leone, D. R. (1994). Facilitating internalization: The self-determination theory perspective. Journal of Personality, 62(1), 119-142.

Deci, E. L., \& Ryan, R. M. (1985). Intrinsic motivation and self-determination in human behavior. New York: Plenum Press.

Deci, E. L., \& Ryan, R. M. (1991). A motivational approach to self: Integration in personality. Paper presented at the Nebraska Symposium on Motivation: Perspectives on motivation, Lincoln, NE.

Deci, E. L., \& Ryan, R. M. (2002). Handbook on selfdetermination research. Rochester, NY: University of Rochester Press.

Edmunds, J., Duda, J. L., \& Ntoumanis, N. (2008). Testing a self-determination theory-based teaching style intervention in the exercise domain. European Journal of Social Psychology, 38, 375-388.

Edmunds, J., Ntoumanis, N., \& Duda, J. (2006). A test of selfdetermination theory in the exercise domain. Journal of Applied Social Psychology, 36, 2240-2265.

Edmunds, J., Ntoumanis, N., \& Duda, J. (2007a). Adherence and well-being in overweight and obese patients referred to an exercise on prescription scheme: A self-determination theory perspective. Psychology of Sport and Exercise, 8, 722-740.

Edmunds, J., Ntoumanis, N., \& Duda, J. (2007b). A test of selfdetermination theory in the exercise domain. In M. S. Hagger \& N. Chatzissaratis (Eds.), Intrinsic motivation and selfdetermination in exercise and sport (Vol. 36, pp. 2240-2265). Champaign, IL: Human Kinetics.

Gagne, M., Ryan, R. M., \& Bargmann, K. (2003). Autonomy support and need satisfaction in the motivation and well-being of gymnasts. Journal of Applied Sport Psychology, 15(4), 372-390

Hair, J. F., Black, W. C., Babin, B. J., \& Anderson, R. E. (2009). Multivariate data analysis $\left(7^{\text {th }}\right.$ ed.). Upper Saddle River, NJ: Prentice Hall.

Hu, L., \& Bentler, P. M. (1999). Cutoff criteria for fit indexes in covariance structure analysis: Conventional criteria versus new alternatives. Structural Equation Modeling: A Multidisciplinary Journal, 6, 1-55.

Kahn, J. H. (2006). Factor analysis in counseling psychology research, training, and practice: Principles, advances, and applications. The Counseling Psychologist, 34, 684-718.

Kennedy, S., Goggin, K., \& Nollen, N. (2004). Adherence to HIV medications: Utility of the theory of self-determination. Cognitive Therapy and Research, 28(5), 611-628.
Kline, R. (2005). Principles and practice of structural equation modeling. New York: The Guilford Press.

Kowal, J., \& Fortier, M. S. (2000). Testing relationships from the hierarchical model of intrinsic and extrinsic motivation using flow as motivational consequence. Research Quarterly for Exercise and Sport, 71, 171-181.

Kubitz, K. (2010). Self-determination theory and exercise behavior in cardiac rehabilitation: A test of mediator variables. Medicine \& Science in Sports \& Exercise, 42(5), 165.

Mardia, K. (1970). Measures of multivariate skewness and kurtosis with applications. Biometrika, 57, 519-530.

Maroco, J. (2010). Análise de equações estruturais: Fundamentos teóricos, software \& aplicações. Pêro-Pinheiro, Portugal: ReportNumber.

Messick, S. (1995). Validity of psychological assessment: Validation of inferences from persons' responses and performances as scientific inquiry into score meaning. American Psychologist, 50, 741-749.

Mireille, J., Renée, L., \& Richard, K. (2008). A SelfDetermination Theory Perspective on parenting. Canadian Psychology, 49(3), 194-200.

Moutão, J., Cid, L., Leitão, J. C., Alves, J., \& Vlachopoulos, S. P. (2012). Validation of the Basic Psychological Needs in Exercise Scale in a Portuguese Sample. Spanish Journal of Psychology, 15(1), 399-409.

Murcia, J., Roman, M., Galindo, C., Alonso, N., \& GonzalezCutre, D. (2008). Peers' influence on exercise enjoyment: A self-determination theory approach. Journal of Sports Science and Medicine, 7, 23-31.

Nunnally, J. C. (1978). Psychometric theory. New York: McGraw-Hill.

Pelletier, L. G., Fortier, M. S., Vallerand, R. J., \& Brière, N. M. (2001). Associations among perceived autonomy support, forms of self-regulation, and persistence: A prospective study. Motivation and Emotion, 25, 279-306.

Reeve, J. (2002). Self-determination theory applied to educational settings. In E. L. Deci \& R. M. Ryan (Eds.), Handbook of self-determination research (pp. 183-203). Rochester, NY: University of Rochester Press.

Reeve, J., Jang, H., Carrell, D., Jeon, S., \& Barch, J. (2004). Enhancing students' engagement by increasing teachers' autonomy support. Motivation and Emotion, 28, 147-169.

Reinboth, M., \& Duda, J. L. (2006). Perceived motivational climate, need satisfaction and indices of well-being in team sports: A longitudinal perspective. Psychology of Sport and Exercise, 7, 269-286.

Ryan, R. M., \& Deci, E. L. (2006). Self-regulation and the problem of human autonomy: Does psychology need choice, self-determination, and will? Journal of Personality, 74(6), 1557-1586.

Ryan, R. M., Patrick, H., Deci, E. L., \& Williams, G. C. (2008). Facilitating health behaviour change and its maintenance: Interventions based on self-determination theory. The European Health Psychologist, 10, 2-5.

Sánchez, J., \& Núñez, J. L. (2007). Análisis preliminar de las propiedades psicométricas de la versión española de la Escala de Necesidades Psicológicas Básicas en el Ejercicio Físico. Revista Iberoamericana de Psicología del Ejercicio y el Deporte, 2, 83-92.

Satorra, A., \& Bentler, P. M. (2001). A scaled difference chisquare test statistic for moment structure analysis. Psychometrika, 66, 507-514.

Silva, M. N., Markland, D., Carraça, E. V., Vieira, P. N., Coutinho, S. R., Minderico, C. S., et al. (2011). Exercise autonomous motivation predicts 3-yr weight loss in women. Medicine and Science in Sport and Exercise, 43(4), 728-737. 
Silva, M. N., Vieira, P. N., Coutinho, S. R., Minderico, C. S., Matos, M. G., Sardinha, L. B., et al. (2010). Using selfdetermination theory to promote physical activity and weight control: A randomized controlled trial in women. Journal of Behavioral Medicine, 33, 110-122.

Steiger, J. H., \& Lind, J. M. (1980). Statistically based tests for the number of common factors. Paper presented at the meeting of the Psychometric Society, Iowa City, IA.

Tobin, V. J. (2003). Facilitating exercise behaviour change: A self-determination theory and motivational interviewing perspective. (Unpublished doctoral dissertation). University of Wales, UK.

Vallerand, R. J. (1989). Vers une méthodologie de validation transculturelle de questionnaires psychologiques: implications pour la recherche en langue française. Psychologie Canadienne, 30, 662-680.

Vansteenkiste, M., Simons, J., Lens, W., Sheldon, K. M., \& Deci, E. L. (2004). Motivating learning, performance, and persistence: The synergistic role of intrinsic goals and autonomy-support. Journal of Personality and Social Psychology, 87, 246-260.

Vincent, J. W. (1995). Statistics in kinesiology. Champaign, IL: Human Kinetics.

Vlachopoulos, S. P. (2007). Psychometric evaluation of the basic psychological needs in exercise scale in community exercise programs: A cross-validation approach. Hellenic Journal of Psychology, 4, 52-74.

Vlachopoulos, S. P. (2008). The Basic Psychological Needs in Exercise Scale: Measurement invariance over gender. Structural Equation Modeling: A Multidisciplinary Journal, 15, 114-135.

Vlachopoulos, S. P., \& Michailidou, S. (2006). Development and initial validation of a Measure of Autonomy, Competence and Relatedness in Exercise: The Basic Psychological Needs in Exercise Scale. Measurement in Physical Education and Exercise Science, 10, 179-201.

Vlachopoulos, S. P., \& Neikou, E. (2007). A prospective study of the relationships of autonomy, competence, and relatedness with exercise attendance, adherence, and dropout. Journal of Sports Medicine Physical Fitness, 47, 475-482.
Vlachopoulos, S., Ntoumanis, N., \& Smith, A. L. (2010). The Basic Psychological Needs in Exercise Scale: Translation and evidence for cross-cultural validity. International Journal of Sport and Exercise Psychology, 8(4), 394-412.

Vlachopoulos, S., \& Triantafyllidou, O. (2008). The contribution of psychological need satisfaction to the prediction of exercise-induced feeling states. Hellenic Journal of Physical Education and Sport Science, 28(4), 10-31.

Williams, G. C., Grow, V. M., Freedman, Z. R., Ryan, R. M., \& Deci, E. L. (1996). Motivational predictors of weight loss and weight-loss maintenance. Journal of Personality and Social Psychology, 70, 115-126.

Williams, G. C., McGregor, H. A., King, D., Nelson, C. C., \& Glasgow, R. E. (2005). Variation in perceived competence, glycemic control, and patient satisfaction: Relationship to autonomy support from physicians. Patient Education and Counseling, 57, 39-45.

Williams, G. C., McGregor, H. A., Sharp, D., Levesque, C. S., Kouides, R. W., Ryan, R. M., et al. (2006). Testing a selfdetermination theory intervention for motivating tobacco cessation: Supporting autonomy and competence in a clinical trial. Health Psychology 25, 91-101.

Williams, G. C., Patrick, H., Niemiec, C. P., Williams, L. K., Devine, G., Lafata, J. E., et al. (2009). Reducing the health risks of diabetes: How self-determination theory may help improve medication adherence and quality of life. Diabetes Educator, 35, 484-449.

Wilson, P. M., \& Rodgers, W. (2004). The relationship between perceived autonomy support, exercise regulations and beahvioural intentions in women. Psychology of Sport and Exercise, 5, 229-242.

Worthington, R. L., \& Whittaker, T. A. (2006). Scale Development Research: A content analysis and recommendations for best practices. The Counseling Psychologist, 34(6), 806-838.

Recebido: 14/01/2011

$1^{a}$ revisão: $28 / 10 / 2011$

Aceite final: 04/11/2011

\section{Anexo A}

Comparação entre a versão original e a traduzida do Perceived Autonomy Support: Exercise Climate Questionnaire (PASECQ)

\begin{tabular}{|c|c|c|}
\hline Item & Versão inglesa (original) & Tradução em Português (versão final) \\
\hline Frase inicial & I feel that my exercise instructor... & sinto que o meu instrutor... \\
\hline Item 1 & ...provides me choices and options. & ...apresenta-me opções de escolha. \\
\hline Item 2 & ... understood me. & ...compreende-me. \\
\hline Item 3 & $\begin{array}{l}\text {...conveyed confidence in my ability } \\
\text { to do well in exercise. }\end{array}$ & $\begin{array}{l}\text {...demonstra confiança na minha capacidade } \\
\text { de realizar os exercícios. }\end{array}$ \\
\hline Item 4 & ...encouraged me to ask questions. & ...deixa-me "à vontade" para colocar questões. \\
\hline Item 5 & ...Listens to how I would like to do things. & $\begin{array}{l}\text {...considera a forma como eu gosto de realizar } \\
\text { os exercícios. }\end{array}$ \\
\hline Item 6 & $\begin{array}{l}\text {...tries to understand how I see things } \\
\text { before suggesting a new way to do things. }\end{array}$ & $\begin{array}{l}\text {...tenta compreender o meu ponto de vista } \\
\text { antes de sugerir algo novo. }\end{array}$ \\
\hline
\end{tabular}

